\title{
Energy Trading from Solar Roof Top
}

\author{
Ariya Phukfon and Suwannee Adsavakulchai \\ Department of Computer Engineering and Financial Technology, University of the Thai Chamber of Commerce, Bangkok 10400, \\ Thailand
}

\begin{abstract}
This research has an objective to study development process of peer-to-peer base simulated energy trading from solar roof top which buyers and sellers can directly offer and purchase electricity energy in closed micro grid distribution system. This study develops the web application for energy trading platform using Microsoft Azure as tool, in addition to developing the Solar Rooftop’s IoT smart device using Arduino Microcontroller Board (MCB) to control relay switching gear for on-grid and off-grid functions and transmit machine status information to web application platform thru mobile frequency's Narrow Band IoT protocol. In conclusion, this study is able to effectively simulate energy trading and feasible for further study in commercialization, whereas, it is necessary to deep-drive study in real environment and other related laws and regulations.
\end{abstract}

Key words: Simulated peer-to-peer energy trading, solar roof top, micro grid, Microsoft Azure, Arduino MCB.

\section{Introduction}

Among major alternative energy types, e.g. wind, geothermal, hydropower, ocean energy, biopower, etc., solar power is recognized as the most suitable energy concerning low carbon emission, reliability and resilience especially in sunny area, ease for installation and maintenance as well as high cost-efficient driving it becomes the most popular clean energy source [1]. While, the average price per watt dropped drastically for solar cells in the decades when the prices in 1977 for crystalline silicon cells were about $\$ 77$ per watt, average spot prices in June 2014 were as low as $\$ 0.36$ per watt or 200 times less than that of almost forty years ago [4]. This price trend was seen as evidence supporting Swanson's law that states that the per-watt cost of solar cells and panels falls by $20 \%$ for every doubling of cumulative photovoltaic production [5]. Economist predicted that solar could contribute to $20 \%$ of total electricity consumption by 2030 [6]. Meanwhile, the cost of energy storages trends to fall sharply than base line prediction since 2013 for $40 \%$ with

Corresponding author: Suwannee Adsavakulchai, Ph.D., associate professor, research fields: computer engineering and artificial intelligence. extent of mass production above $\$ 300 / \mathrm{kW}$ (base line prediction was $\$ 500 / \mathrm{kW}$ in 2020) [7]. With compound of both cheaper crystalline silicon cells and energy storages, its total cost is predicted to reach hurdle to beat the conventional fuel base electricity by 2021 [8]. At the end of 2015, Thailand, with a total capacity of 2,500-2,800 MW, share exceeds $60 \%$ of total installed capacity in Association of Southeast Asian Nations (ASEAN) [9] meaning it has more solar power capacity than all the rest of ASEAN countries combined targeting to reach 6,000 MW for reducing $65 \%$ of gas-fired electricity mix to $40 \%$ by $2036[8,9]$.

In order to develop the simulated peer-to-peer energy trading in micro grid from solar rooftop, this study aims to develop a web application platform for sending electricity demand order and electricity supply transmission, so-called energy trading, using Microsoft Azure platform and Solar Rooftop's industrial internet of things (IoT) smart device using Arduino microcontroller board to control relay switching gear for on-grid and off-grid functions and transmit machine status information to web application platform thru mobile frequency's Narrow Band (NB) IoT protocol. 


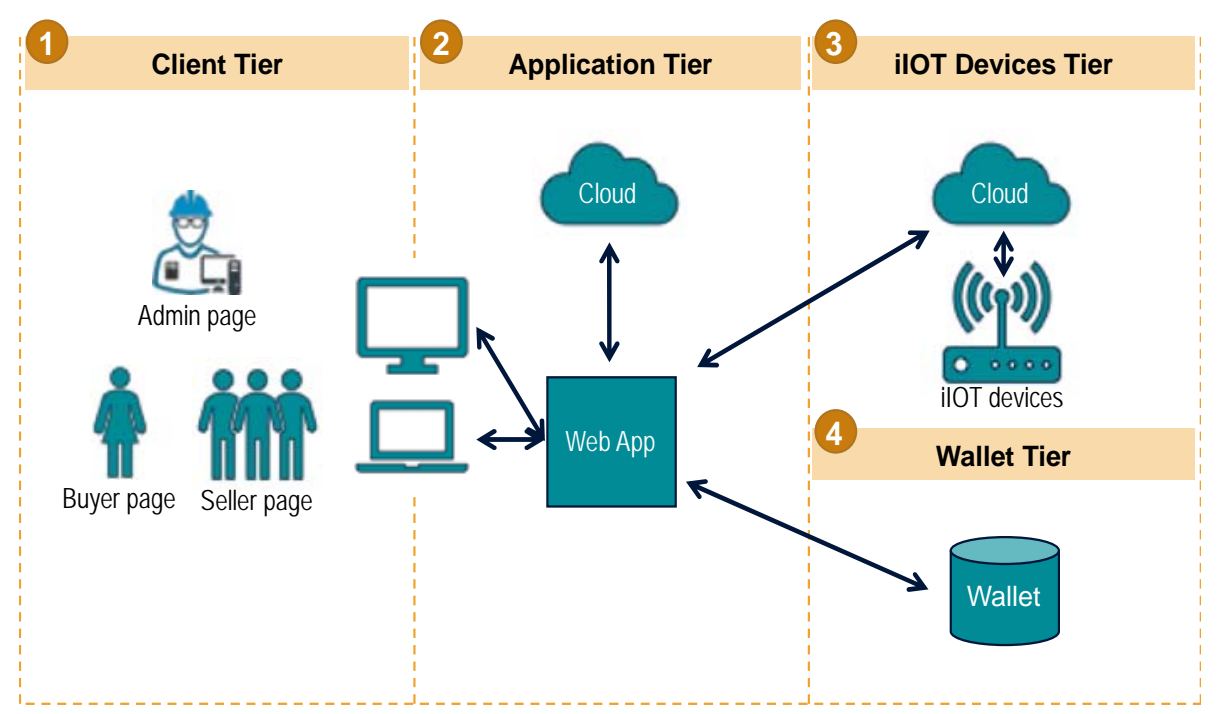

Fig. 1 Development modules.

\section{Research Methodology}

The research framework is based on the agile approach for each development phase which is incrementally added to the total platform. New or updated features were added by rerunning small sprints. There are 4 development modules i.e. Client Tier, Application Tier, iIoT Devices Tier, and Wallet Tier as shown in Fig. 1.

From Fig. 1, the activities of each module are following.

\subsection{Client Tier}

It is to develop the web application by designing the user interface (UI) for home page/admin page including the marketplace, buyer and seller page and Grid operator page.

\subsection{Application Tier}

It is to develop the energy trading model and application programming interface (API) including all services.

\subsection{IIot Devices Tier}

It is to develop switching gate control, electricity powers measuring and the linkage between Arduino Microcontroller Board (MCB) and Narrow Band IoT (NB-IoT).

\subsection{Wallet Tier}

It is to develop the simulation of admin, buyer and seller wallet.

\section{Identify Business Requirement}

Comparing to typical trading activities in other businesses, the energy trading has key different points in order to deliver business success as below.

- Electricity unit sale has 3 dimensions including electricity power $(\mathrm{kW})$, frequency $(\mathrm{Hz})$, and time (h) while typical trading commodities have only 2 dimensions as size or volume and weight.

- Electricity is one of the critical limited resources so that it has to control demand and supply of the market called Demand Side Operation (DSO) while typical trading commodities are controlled by demand and supply of the market in liberalized economic environment.

- Reliability and efficiency are highly required. The prompt demand response (DR) is used to measure quality of electricity supply called the System Average Interruption Duration Index (SAIDI) and The System Average Interruption Frequency Index (SAIFI).

- In nowadays electricity energy system, the electricity is limited to wired transfer that has to involve public or private grid operators and regulators. 
- Electricity market regulations may differ from countries to countries and markets to markets whose regulators in Thailand have tightly centralized control over this market.

Hence, this study described not only business requirement but also relationship with grid operators and regulators. However, relevant web application features were not included.

\subsection{Simulated Energy Trading}

The simulated energy trading consists of single buyer and 2 sellers which both buyer and sellers possess at least one smart device that has functionality similar to smart meter.

\subsection{Overall Business Requirement}

There are 5 steps in business requirement: (1) to declare who is willing to buy and available to sell; (2) to do matching the demand and supply; (3) to seek approval from operator; (4) to start energy trading and (5) finally to process in energy trading.

\section{Design the Prototype}

In this study, there are six prototypes as follows.

\subsection{Cloud Platform Selection}

Select the 4 key features as minimum requirement for the web application consisting of Azure cloud service, Azure SQL database, web applications, and notification hub because it was very easy and efficient to develop frontend to backend application. While True Corp's NB IoT is to connect with Google's IoT cloud thru CoAP, it was convenient to set up as well as high data transmission rate. Both cloud platforms made connection with application program interfaces (APIs).

\subsection{Programming Language Selection}

Select the computer programs as C\#, Java Script, REST API, MSSQL, Node. JS, Microsoft. Asp Net Core. Http, and Microsoft. Entity Framework Core. Metadata for developing the web application because it was easy to use with Microsoft Azure.

\subsection{Microcontroller Board, Sensors and Switch} Selection

Select Arduino UNO R3 and Lambda Basic v1.3 for MCB because it has functionality and capability with high cost-efficiency which Arduino IDE language was used for programming the MCB. For measuring electricity current, select the ACS712 Current Sensor Module 30A Model Arduino compatible while selecting 10A 1 Channel Arduino Compatible Relay switch because it is durable for electricity current to be used to simulate the energy trading.

\subsection{Standard Measurement Device for Sensors Calibration}

Select multimeter Uni-T UT203 Digital Handheld Clamp multimeter DMM DC/AC Volt Amp Tester Meter for calibrating the sensors.

\subsection{Battery Selection}

Select the battery cycle with the maximum's $12-\mathrm{V}$ 1.3AH, Charge controller Kranchana Electric's 30A $12 \mathrm{~V} / 24 \mathrm{~V}$, and Invertor Foval's DC12V to AC 230V 2000W to simulate solar energy management device.

\subsection{Wireless Device Selection}

Select the wireless data transmission device True Corp's NB IoT board (shield for Arduino) module Quectel BC95-B8 using $900 \mathrm{MHz}$ band because solar rooftop may be installed in remote area which is difficult for maintenance and where the NB IoT has wider coverage, lower power consumption, and longer longevity than typical LAN and Wi-Fi.

\section{Development of the Prototype Platform}

\subsection{Software Development}

Develop the platform using the agile method for building up modules with small sprints starting from trading module and trading database to customer management module and e-wallet. An energy trading 
marketplace covers $\mathrm{B} 2 \mathrm{~B}, \mathrm{~B} 2 \mathrm{C}$ and $\mathrm{P} 2 \mathrm{P}$ method as well as in connectivity with iIoT devices for ensuring transparent, secure, fast and cheap transaction. E-Wallet platform is the platform for managing and settling payment for each transaction. Then use Swagger UI-API to simulate the smart devices as shown in Fig. 2. By using model-view-controller (MVC) concept, researchers developed system architecture for platform development shown in Fig. 2.

\subsection{Hardware Development}

In order to simulate energy trading activity, prototype of 3 sellers and single buyer was developed shown in detail diagram as Fig. 3, so-called smart device. The smart device has functionality not only to control the switching relay but also able to send and receive information to software application necessary in energy trading activity.

\subsection{Connection between Software and Hardware Platform}

Test and retest the connection between software and hardware platform along the experiment while adjusting the connection along the way as well.

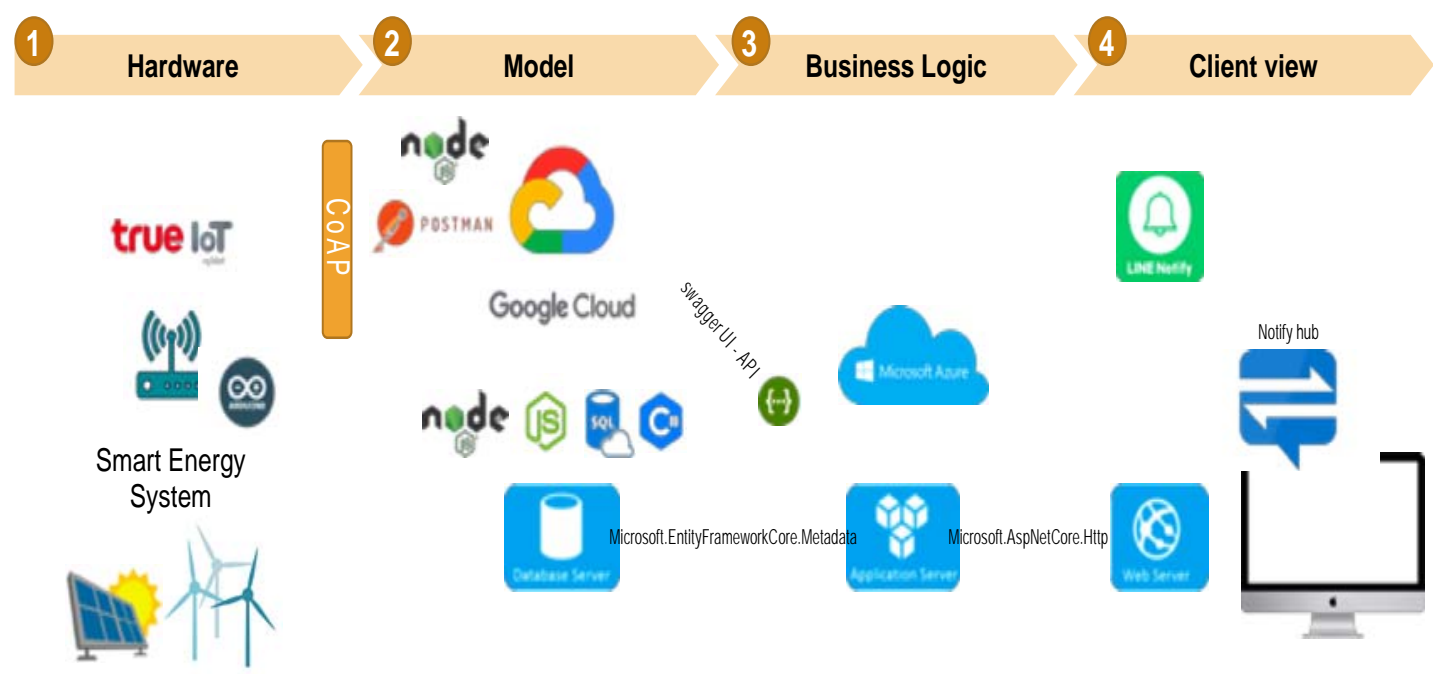

Fig. 2 Platform development method.

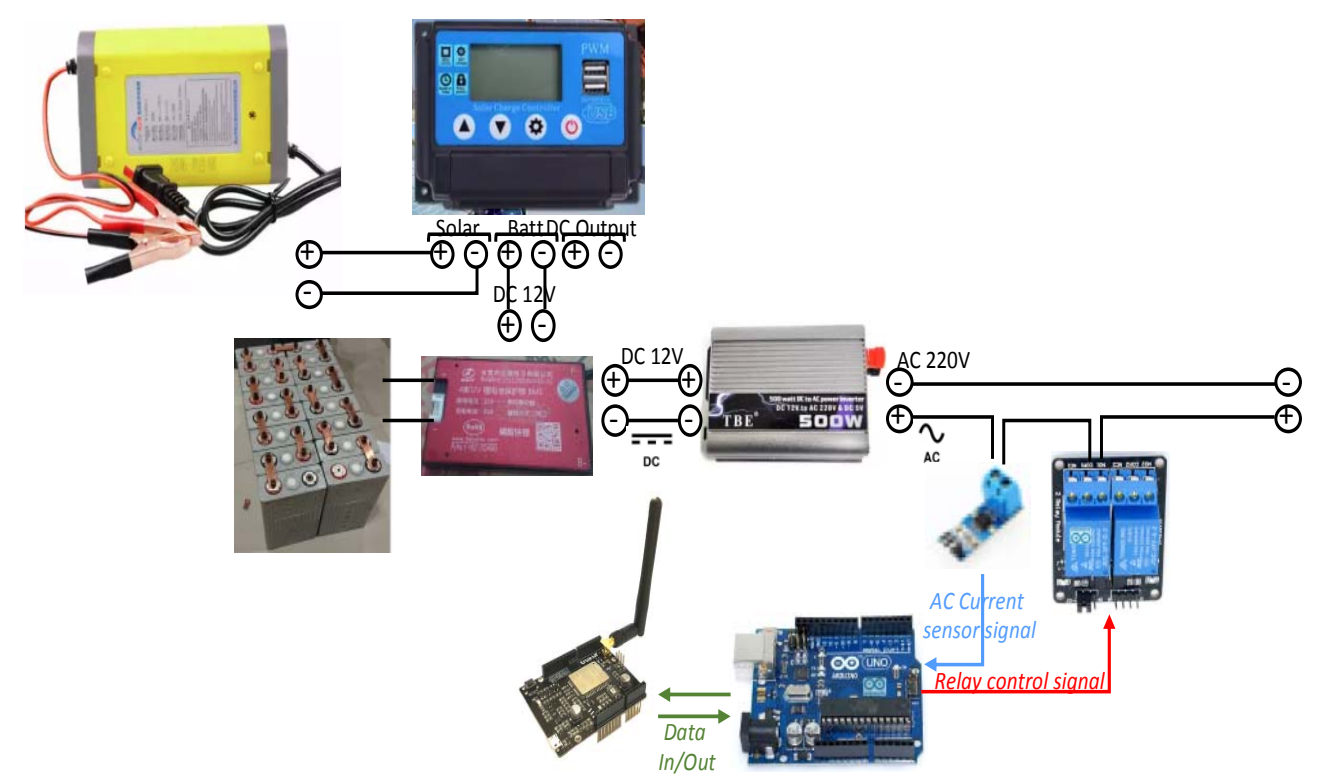

Fig. 3 Hardware diagram. 


\section{Results and Discussion}

\subsection{Web Application}

There are 6 functionalities for energy trading as follows.

- The Home page provides Home/Login functionality while it also provides generic information of the project such as project concept, about us, and about energy trading.

- The User registration page provides the Register energy item functionality that buyer and seller have to provide individual/organization information, e.g. name-surname, address, contact information, address, GPS location as well as machine information, e.g. smart meter serial number, invertor IP address, capacity etc.

- The Energy trading page provides Display/Browses energy trading, select energy items, order energy items, and transfer energy functionalities that sellers can post to sell their energy capacity with proposed price per unit and buyers can select capacity to buy from whom at proposed unit price by issuing purchase order. The energy trading started to count down for transferring energy once both parties mutually agreed on the transaction.

- The Wallet page provides the Notify transfer completion and Settle payment functionality, which displays individual account balance information and transaction ledger. The value amount represents Thai baht fiat currency by which settlement of the trading executes with web application algorithm.

- The User transaction dashboard provides personal view of energy trading transaction's summary information.

- The Transaction history page provides the Notify transfer completion and Settle payment functionality, which displays selling and purchasing transaction data.

\subsection{Simulated Energy Transmission Stage}

Energy trading method occurs in real possible energy trading in micro grid which is able to identify 4 stages as shown in Fig. 4.

\section{Stage 1: All off}

No electricity current supplied to the simulated system. Light was turned off.

Stage 2: Closed grid (off grid)

Electricity from single solar rooftop source supplied only to the simulated system. Light was turned on with low brightness.

Stage 3: Plug to public grid (on-grid)

Electricity from single solar rooftop and public electricity distribution source supplied to the simulated system. Light was turned on with normal brightness.

Stage 4: Free trade

Electricity from 2 solar rooftops and public electricity distribution source supplied to the simulated system. Light was turned on with high brightness.

\subsection{Integration between Hardware and Software}

The connection between web application and iIoT smart device by choosing Enhanced Independently Self-Management Model enhanced the smart device to independently execute necessary command so that the smart device can operate based on self-reliance as well as reduce connection to traffic and energy consumption.

\subsection{Discussion}

Command orders to control light bulb for turning on/off were synchronously sent to both experiment kits at the same time with 2 testing modes as normal and flashing mode. Researcher found that transmitting via NB IoT had longer responding time by approximately 1-2 seconds than others in normal mode. When testing with flashing mode, command order's responding time for all connection method took longer and longer so that researcher changed data storage from MCB's RAM to cloud and then the issue is solved. 


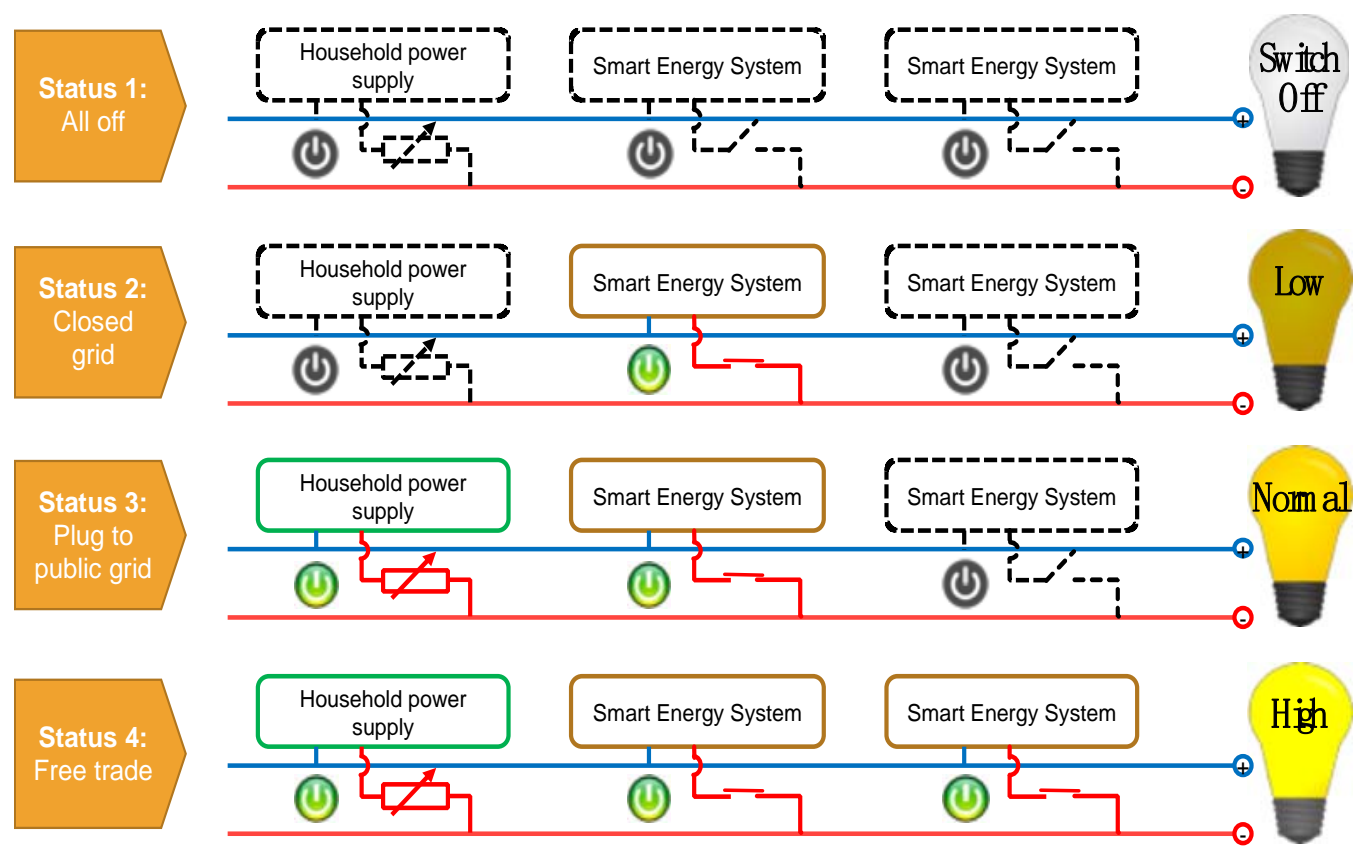

Fig. 5 Simulated energy transmission stage.

\section{Conclusion and Recommendation}

\subsection{Conclusion}

This study proposed agile method to develop energy trading platform using Microsoft Azure that is able to offer peer-to-peer energy trading from sellers to buyers and industrial IoT able to transmit solar rooftop and system data as well as receive command from platform to manage relay switch hardware via NB IoT. The proof of concept shows system capability to organize the energy trading from both software and hardware perspective which is feasible to study further for commercialization while it still needs to develop related software, hardware, and iIoT smart device to reach scalable system as well as explore legal constraints. After several trial, adjustment, and troubleshooting, the platform can successfully simulate energy trading using the web application and iIoT smart device.

\subsection{Recommendation}

This web application can be able to explore further advance technology such as blockchain and machine learning for reaching smarter platform. In order to commercialize, the web application and iloT smart device shall increase to be scalable platform with effective cyber resilience.

\section{References}

[1] The Economist. 2012. "Sunny Uplands: Alternative Energy Will No Longer Be Alternative.” http:// www.economistcom/news/2012/11/21/sunny-uplands.

[2] Michael Graham Richard via Bloomberg New Energy Finance. 2015. "This Striking Chart Shows Why Solar Power Will Take Over the World.” https://www.treehugger.com/renewable-energy/striking-c hart-showing-solar-power-will-take-over-world.html.

[3] Ho, E. 2017. "Solar Power Development in Southeast Asia.” https://asian-power.com/regulation/commentary/ solar-power-development-in-southeast-asia.

[4] Jittapong, K. 2015. “Thailand Ignites Solar Power Investment in Southeast Asia.” https://www.reuters.com/ article/thailand-solar/thailand-ignites-solar-power-invest ment-in-southeast-asia-idUSL3N0ZM2JB20150712.

[5] Kingdom of the Netherlands. 2017. "Solar Power in Thailand.” https://www.rvo.nl/sites/default/files/2017/03/ FACTSHEET-SOLAR-POWER-IN-THAILAND.pdf.

[6] The Guardian. 2014. "Are Solar Farms Really Hitting British Food Production?” https://www.theguardian.com/ environment/2014/oct/21/are-solar-farms-really-hitting-br itish-food-production.

[7] Business Insider. 2017. "You Can Officially Order Tesla's Solar Roof-Here's Everything You Need to Know.” 
https//:www.business insider.com/tesla-solar-roof-ordersopen-features -photos-2017-5.

[8] Bangkok Post. 2017. "Smart Grids on Cards in PEA 4.0 Drive.” https://www.bangkokpost.com/business/news/ 1221862/smart-grids-on-cards-in-pea-4-0-drive.

[9] Zhang, C., and Associates. 2017. "Review of Existing Peer-to-Peer Energy Trading Projects.” https://orca-mwe. cf.ac.uk/111424/1/1-s2.0-S1876610217308007-main.pdf. 